\title{
Resounding failure to replicate links between developmental language disorder and cerebral lateralisation
}

\author{
Alexander C Wilson ${ }^{\text {Corresp., }}{ }^{1}$, Dorothy V M Bishop ${ }^{1}$ \\ ${ }^{1}$ Department of Experimental Psychology, University of Oxford, Oxford, United Kingdom \\ Corresponding Author: Alexander C Wilson \\ Email address: alexander.wilson@psy.ox.ac.uk
}

Background: It has been suggested that failure to establish cerebral lateralisation may be related to developmental language disorder (DLD). There has been weak support for any link with handedness, but more consistent reports of associations with functional brain lateralisation for language. The consistency of lateralisation across different functions may also be important. We aimed to replicate previous findings of an association between DLD and reduced laterality on a quantitative measure of hand preference (reaching across the midline) and on language laterality assessed using functional transcranial Doppler ultrasound ( $\mathrm{FTCD}$ ).

Methods: From a sample of twin children aged from 6;0 to 11;11 years, we identified 107 cases of DLD and 156 typically-developing comparison cases for whom we had useable data from fTCD yielding a laterality index (LI) for language function during an animation description task. Handedness data were also available for these children.

Results: Indices of handedness and language laterality for this twin sample were similar to those previously reported for single-born children. There were no differences between the DLD and TD groups on measures of handedness or language lateralisation, or on a categorical measure of consistency of left hemisphere dominance. Contrary to prediction, there was a greater incidence of right lateralisation for language in the TD group (19.90\%) than the DLD group (9.30\%), confirming that atypical laterality is not inconsistent with typical language development. We also failed to replicate associations between language laterality and language test scores.

Discussion and Conclusions: Given the large sample studied here and the range of measures, we suggest that previous reports of atypical manual or language lateralisation in DLD may have been false positives. 
1 Resounding failure to replicate links between developmental language disorder and cerebral

2 lateralisation

\author{
Alexander C. Wilson ${ }^{1} \&$ Dorothy V. M. Bishop ${ }^{1}$ \\ ${ }^{1}$ Oxford University
}

3

4

\title{
Author note
}

5 Correspondence concerning this article should be addressed to Alexander C. Wilson,

6 Department of Experimental Psychology, South Parks Road, Oxford, UK, OX1 3UD. E-mail:

$7 \quad$ alexander.wilson2@psy.ox.ac.uk

\section{Abstract}

9 Background: It has been suggested that failure to establish cerebral lateralisation may be 10 related to developmental language disorder (DLD). There has been weak support for any link 11 with handedness, but more consistent reports of associations with functional brain lateralisation

12 for language. The consistency of lateralisation across different functions may also be important.

13 We aimed to replicate previous findings of an association between DLD and reduced laterality on

14 a quantitative measure of hand preference (reaching across the midline) and on language

15 laterality assessed using functional transcranial Doppler ultrasound (fTCD).

16 Methods: From a sample of twin children aged from 6;0 to 11;11 years, we identified 107

17 cases of DLD and 156 typically-developing comparison cases for whom we had useable data

18 from fTCD yielding a laterality index (LI) for language function during an animation description

19 task. Handedness data were also available for these children.

20 Results: Indices of handedness and language laterality for this twin sample were similar to 21 those previously reported for single-born children. There were no differences between the DLD 22 and TD groups on measures of handedness or language lateralisation, or on a categorical measure 
23 of consistency of left hemisphere dominance. Contrary to prediction, there was a greater

24 incidence of right lateralisation for language in the TD group (19.90\%) than the DLD group

$25(9.30 \%)$, confirming that atypical laterality is not inconsistent with typical language development.

26 We also failed to replicate associations between language laterality and language test scores.

27 Discussion and Conclusions: Given the large sample studied here and the range of

28 measures, we suggest that previous reports of atypical manual or language lateralisation in DLD

29 may have been false positives. 


\section{Resounding failure to replicate links between developmental language disorder and cerebral lateralisation}

\section{Introduction}

The relationship between atypical brain lateralisation and developmental language disorder (DLD) has intrigued scientists for many years, but is still not well understood. Lateralisation is thought to reflect an adaptive process of specialization by which cognitive functions become preferentially supported by one cerebral hemisphere: in the case of language, typically the left hemisphere. Theoretical accounts have suggested that individuals who do not show the population bias towards left hemisphere dominance for language may be at risk of disrupted language development (e.g. Annett, 2002; Bishop, 2013; T. J. Crow, Crow, Done, \& Leask, 1998).

The earliest studies to test the relationship between hemispheric dominance and language problems measured laterality by handedness. Handedness has been used as a marker of language lateralisation given a link between the two traits: left-handers are more likely than right-handers to have atypical lateralisation for language (Knecht et al., 2000; Szaflarski et al., 2002). If atypical laterality is associated with language and literacy disorders, then the expectation is that more left-handers will have these disorders. While handedness assessed via a questionnaire inventory has shown no link with speech and language problems (Bishop, 2001, 2005), a relationship has been reported for reduced right hand preference in a task requiring reaches across the midline (Bishop, 2005; E. L. Hill \& Bishop, 1998). With respect to literacy, a meta-analysis indicated a significant over-representation of left-handers among those with dyslexia (Eglinton \& Annett, 1994). However, this meta-analysis did not weight the effects of individual studies for their sample size and did not calculate a summary effect size, so the reported relationship is difficult to interpret. Importantly, there are also numerous large epidemiological studies failing to find any evidence of a link between handedness and reading problems (e.g. Levinson, 1988; Rutter \& Yule, 1970; Satz \& Fletcher, 1987). Investigation of handedness as a predictor of language ability in typically developing individuals provides further uncertain (probably null) results. For instance, a meta-analysis found no relationship in the full analysis $(\mathrm{N}=359,890)$, and 
62 a very small disadvantage for left-handers (Hedge's $g=-0.09)$ when only children were analysed

63 (M. Somers, Shields, Boks, Kahn, \& Sommer, 2015). Methodologically, there is concern in the

64 field that flexible criteria for categorisation of handedness, as well as selective reporting of results

65 only when a significant effect is found, may have led to inflated type 1 error (Bishop, 1990).

66 Overall, the evidence provides weak grounds for predicting that DLD is related to reduced right

67 handedness.

68

69
It is important to note, however, that manual laterality is at best a weak proxy for language lateralisation in the brain (Groen, Whitehouse, Badcock, \& Bishop, 2013). A smaller literature using more direct brain measures of language laterality does appear to support the view that there may be reduced left hemisphere dominance in those with DLD. Small-scale studies using functional transcranial Doppler sonography (fTCD) have compared task-related blood flow in the middle cerebral arteries (MCAs) during productive language paradigms. Illingworth and Bishop (2009) found reduced left lateralisation in a sample of dyslexic adults $(n=30)$ compared to controls, while Bishop, Holt, Whitehouse, and Groen (2014) reported that four year-olds with language problems $(n=11)$ did not have significantly left-lateralised language function at the group level, whereas those with typically developing language showed the usual left bias found in adults. Compared to typical controls, Whitehouse and Bishop (2008) indicated that a pattern of right and bilateral distribution of language function characterised adults with persisting specific language impairment $(n=11)$, whereas typical laterality was found for those whose language problems had resolved $(n=9)$ and a group with autism spectrum disorder $(n=11)$. This laterality difference between those with autism and those with language difficulties chimes with Lindell and Hudry's (2013) literature review of language lateralisation in autism, which provided the strongest evidence for atypical laterality in individuals with ASD who also had comorbid language difficulties. This supports the view that atypical laterality is relevant to other neurodevelopmental disorders but particularly implicated in DLD.

Functional MRI studies, in which blood oxygenation levels during language tasks are compared to a baseline, corroborate the fTCD findings reported above. Thus, reduced left laterality was found in two small samples of children with specific language impairment $(n=21$; $\mathrm{n}=10$ ) compared to controls (Badcock, Bishop, Hardiman, Barry, \& Watkins, 2012; de Guibert et al., 2011). A further study reported greater right lateralisation in children with speech delay (n 
92 = 17) compared to controls (Bernal \& Altman, 2003), though statistical significance was only

93 reached in the latter study when decomposing the sample by age. Laterality indices from fMRI

94 studies of individuals with dyslexia have also indicated reduced left laterality (Waldie, Haigh,

95 Badzakova-Trajkov, Buckley, \& Kirk, 2013; Xu, Yang, Siok, \& Tan, 2015), though the clinical

96 samples again were small ( $\mathrm{n}=12$ in both). In addition, structural MRI studies provide some

97 evidence for atypical structural asymmetries, especially in frontal language regions, in children

98 with language disorder, as reviewed by Mayes, Reilly and Morgan (2015). This review presented

99 limited evidence for the hypothesis that typical cerebral asymmetries are disrupted in language

100 disorder.

101 Research assessing whether cerebral laterality predicts language skills in typically

102 developing individuals provides some further evidence that left hemisphere dominance is

103 advantageous. A moderate positive relationship has been reported between left lateralisation and

104 vocabulary and non-word reading skills using fTCD $(n=55)$ (Groen, Whitehouse, Badcock, \&

105 Bishop, 2012); a trend with word reading did not meet significance. Meanwhile, Everts et al.

106 (2009) found a large correlation $(r=.59)$ between verbal IQ and left lateralisation in a fMRI

107 vowel detection task in a sample of 20 adolescents; the correlation with laterality derived from a

108 fMRI synonym decision task was moderate in size but not statistically significant. In 24 young

109 adults, left lateralisation of Wernicke's area during fMRI productive language tasks was part of a

110 principal component also including greater functional connectivity at rest and greater symmetry

111 of the arcuate fasciculus that predicted verbal IQ $(r=70)$ (Piervincenzi et al., 2016). An

112 interesting counterpoint to these findings is an fMRI study of language lateralisation in a sample

113 of patients with callosal agenesis $(n=25)$ (Hinkley et al., 2016). While there was no correlation

114 between laterality and verbal IQ in 21 healthy matched controls, there was a high correlation in

115 the patients $(r=.55)$. The lack of relationship in the healthy controls contradicts other findings,

116 though the restricted variance in the group may explain this. However, the effect reported for the

117 patients suggests that where normal lateralisation processes are disrupted, the recruitment of the

118 left hemisphere for language is most adaptive for language development.

119 However, results have not been unambiguous in their support of the hypothesis that DLD is 120 associated with atypical language lateralisation. For instance, Berl et al. (2014) found non-

121 significant correlations between several language measures and left lateralisation of Wernicke's 
122 area in a sample of 4-12 year-olds $(n=56)$, contrary to the effects reported in other studies listed

123 above - though there was a significant correlation with lateralisation of the cerebellum.

124 Counterintuitively, right-lateralised language-related activity in the cerebral cortex has not always

125 been reported as detrimental for language development. Thus, Ettinger-Veenstra et al. (2010)

126 reported a relationship between more right lateralised language activity and better performance

127 on neuropsychological tests of language and reading ability ( $r=$ around -.5$)$ in 14 healthy adults.

128 In a large study that oversampled left handers (153 in a total sample of 297), individuals with

129 strong hemispheric dominance for language (whether left or right) showed slightly stronger

130 performance than those with more symmetrical language laterality on verbal, spatial and verbal

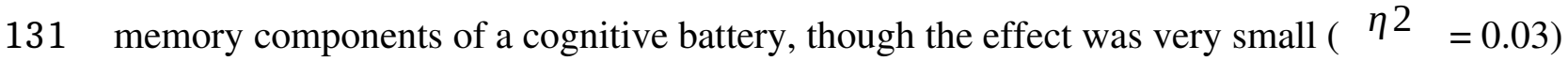

132 (Mellet et al., 2014). Thus, evidence is mixed, but we can infer from this unusually large fMRI

133 study that atypical laterality does not necessarily entail a cognitive disadvantage. Some atypically

134 lateralised individuals clearly perform above average on verbal and non-verbal assessments.

135 Given this lack of a simple link between laterality and language skills, it is possible that a

136 more complex relationship exists between the two. For one thing, it need not be assumed that all

137 language functions show the same pattern of lateralisation within the individual, especially given

138 that several networks seem to be implicated in different aspects of language processing

139 (Friederici, 2011). Bishop (2013) proposed that the lateralisation of different aspects of language

140 processing may show one of two endophenotypes in the individual: a left-brain bias that

141 promotes left-hemisphere mediation of all language functions vs an unbiased brain, where there

142 is equal likelihood of different language functions developing in the left or right hemisphere.

143 Given the partial relationship between language laterality and handedness (e.g. Knecht et al.,

144 2000), these endophenotypes may also apply to manual laterality, with the left-brain bias

145 promoting left hemisphere dominance for motor functions and the unbiased brain leaving

146 handedness to chance. This model is in line with earlier genetic models in which the recessive

147 allele of a hypothetical "laterality gene" was thought to remove the typical bias for both left

148 language lateralisation and right-handedness (McManus, 1985; Annett, 2002). Current research

149 rejects the hypothesis that language lateralisation and handedness are controlled by a single gene

150 (Ocklenburg, Beste, Arning, Peterburs, \& Güntürkün, 2014), but there is evidence that the "no

151 bias" endophenotype for handedness predicts a similar "no bias" endophenotype for language 
152 lateralisation, as these earlier theories proposed. This evidence includes two fMRI studies of

153 monozygotic twins that found significant concordance for language lateralisation only within

154 twin pairs concordant for handedness (Badzakova-Trajkov, Haberling, \& Corballis, 2010;

155 Sommer, Ramsey, Mandl, \& Kahn 2002). Twins discordant on the laterality measures provide

156 evidence for the existence of a "no bias" endophenotype in which laterality develops randomly

157 within twins and genetic control over laterality is lost. The presence of this "no bias"

158 endophenotype predicts that overall heritability of laterality across the population should only be

159 modest, and empirical data support this. In a study of 368 people from 37 families, handedness

160 heritability was 0.24 and language lateralization by fTCD was 0.31 - and it is worth noting that

161 this may be an overestimation given that families were oversampled for cross-generational

162 sinistrality (Somers et al., 2015).

163 The left-brain bias model assumes that the bias operates separately and in a probabilistic

164 fashion for different functions. A person with left-brain bias will tend to have all language-related

165 functions mediated preferentially by the left hemisphere, and is likely to show right handedness.

166 A person with no bias is more likely to have discrepant lateralisation for different functions, and

167 this may increase the risk of developmental language problems. According to this model, a single

168 measure of lateralisation will give only a crude indication of whether a person is in the left-bias

169 category. However, where individuals show consistent left laterality on different measures of

170 language laterality, and potentially on measures of handedness too, this is likely to indicate that

171 they are of the left-bias endophenotype, which may be protective against language problems.

172 Based on this model and existing research on laterality and DLD, we hypothesise (a) that reduced

173 left lateralisation is associated with DLD, and (b) that discrepancies in laterality across measures

174 of language lateralisation and handedness is a risk factor for DLD.

175 In the current study, we aimed to test the left-brain bias model, using data from a sample of 176 twin children who had been assessed on language and literacy skills, as well as on two measures 177 of handedness and a direct measure of cerebral lateralisation for language. The sample had been

178 selected to be over-representative of cases of DLD. In the current paper, the relationship between

179 language and laterality is probed, with the twin status of the children taken into account using

180 multi-level modelling. In a related paper, we consider heritability of laterality assessed by

181 comparing monozygotic and dizygotic twins. 
We report how we determined our sample size, all data exclusions, all manipulations, and

184 all measures in the study.

\section{Participants}

186 We recruited families with twin children aged between $6 ; 0$ and 11;11 years, whose first

187 language at home was English. We aimed for an over-representation of twin pairs in which one or

188 both twins had language or literacy problems that might be indicative of DLD. Families were

189 recruited via fliers sent to primary schools around the UK, advertisements on our group's website

190 and via twins' clubs. The initial flier was worded as follows: 'We are looking for sets of twins to

191 participate in a new study investigating factors underlying children's language difficulties. We

192 want to test twins with and without language problems (language-impaired, typically-developing,

193 or one twin of each).' Head teachers were asked to forward information sheets about the study to

194 parents of twin children. We aimed to recruit 180 pairs selected on the basis of having language

195 or literacy problems (60 MZ, 60 DZ opposite sex and 60 DZ same sex), and 60 unselected pairs

196 (20 of each type). In practice, self-selection of those volunteering to take part meant that the latter

197 group tended to come from relatively highly educated backgrounds, and could not be regarded as

198 representative of the general population. The flow chart in Fig. 1 shows the numbers of

199 participant children at different stages of selection. Parents and caregivers of 194 twin pairs

200 volunteered for the study, yielding 134 children who met our criteria for DLD, and 190 children

201 who met criteria as typically developing (TD). See the Data Analysis section below for criteria.

\section{INSERT FIGURE 1}

Children were excluded from the sample if they met any of the following criteria: WASI 204 nonverbal ability (performance IQ) more than two SDs below the population mean; diagnosis of 205 autism spectrum disorder (ASD) in one or both twins; sensorineural hearing loss or failure of a 206 hearing test on the day of testing; and brain injury or a serious medical condition affecting one or 207 both twins. In order to test our main hypothesis that DLD was related to cerebral laterality as 208 measured by fTCD, it was necessary to exclude individuals in a second stage of exclusions if we 209 did not obtain useable fTCD data from them, defined as fewer than 12 accepted trials. We also 
210 excluded participants with extreme laterality indices (above 10 or below -10); 3 individuals were

211 excluded based on this criterion. Useable fTCD data were obtained from 107 (80\%) of the

212 children with DLD, and $156(82 \%)$ of the TD children.

\section{Material}

\section{Language, literacy and cognitive assessments}

214 The assessment battery used to categorise language status is shown in Table 1.

Nonverbal ability was estimated using the two nonverbal subtests of the WASI (Block

217 Design and Matrices); age-normed scores on these were converted into an overall Performance

218 IQ score. The remaining tests of the battery were used to index language and literacy abilities. All

219 measures involved individual assessment by a trained examiner, except for the CCC-2, which is a 220 parental report instrument.

221 Laterality assessments Handedness. Handedness was assessed using the same hand 222 preference battery as in Bishop (2005). This was based on items from the Edinburgh Handedness 223 Inventory (EHI) (Oldfield, 1971), modified to replace one item (striking a match) deemed 224 unsuitable for children. The experimenter asked the child to demonstrate how they would 225 perform each of the following actions: writing, drawing, throwing a ball, using scissors, using 226 toothbrush, cutting with a knife, using a spoon, using a broom, taking the lid off a box, and 227 dealing cards. One point was awarded for exclusive right hand use, zero points for left hand use, 228 and half a point if both hands were used, giving a score ranging from zero to ten. This score was 229 then converted to a laterality index ranging from -100 (extreme left handedness) to 100 (extreme 230 right handedness). In addition to this score, a child was categorised as right-handed if they scored 231 above 0 on this measure.

232 Quantitative Hand Preference. A measure of strength of hand preference was obtained 233 from the second measure of handedness, the Quantification of Hand Preference (QHP) task 234 (Bishop, Ross, Daniels, \& Bright, 1996). This measures an individual's tendency to continue to 235 use the preferred hand when items are placed across the midline. In this task, the child stands in 
236 front of a semi-circular array of picture cards, with three cards in each of seven positions

237 extending at 30-degree intervals from the left to the right of the child's midline. The child is asked

238 to pick up a named card and place it in a central box. The child is not told that handedness is

239 being assessed, and no instructions are given about how to handle the cards or how to stand, other

240 than that to remain in the central location in front of the box. The same quasi-random order of

241 positions is used for all children, starting with a card at the midline and continuing until the child

242 has reached for three cards at each of seven locations. One point was recorded for each right-

243 handed reach, giving a possible total of 21 . In addition to this quantitative score, a child was

244 categorised as right-handed by this measure if they scored over 10 points.

$245 \quad$ Language laterality. Language laterality was assessed using functional transcranial

246 Doppler ultrasound (fTCD) while the child performed a productive language task. Transcranial

247 Doppler ultrasound is a technique used in medical contexts to assess the integrity of the cerebral

248 blood vessels using ultrasound probes placed on the temples. In this study, probes were attached

249 to a headset and positioned to detect blood flow in the left and right middle cerebral arteries

250 (MCAs), which supply language-relevant regions in the lateral aspects of the frontal, temporal

251 and parietal lobes. Researchers conducting the procedure were trained to identify the blood

252 vessels, which have distinctive characteristics in terms of depth and direction of flow.

253 The language task used was the animation description paradigm, for which a video

254 demonstration can be accessed from Bishop, Badcock, and Holt (2010), and which was

255 implemented with children by Groen et al. (2012). On each trial, the child silently views a $12 \mathrm{~s}$

256 clip from a cartoon including sounds but no speech. A response cue then indicates the start of a

$25710 \mathrm{~s}$ talk phase during which the child is asked to describe what happened in the cartoon. A

258 second cue then indicates that the child should stop talking. This paradigm has previously been

259 found to have good validity and reliability (Bishop, Watt, \& Papadatou-Pastou, 2009). A

260 maximum of 30 trials was administered, depending on the child's tolerance of the procedure. The

261 child's verbal responses were recorded and subsequently transcribed, and the examiner noted

262 behaviour during the procedure. Trials were excluded where the child either spoke during a silent

263 period, or failed to talk during the talk phase: these infringements need to be omitted because

264 they invalidate analysis of the trial, which involves comparing cerebral blood flow during the

265 period of interest when the child talks with a baseline period when no talking occurs. The 
266 baseline is taken to be the $12 \mathrm{~s}$ spent watching the animation immediately before the talk phase 267 and the period of interest commences $4 \mathrm{~s}$ into the talk phase and lasts for $10 \mathrm{~s}$. The $4 \mathrm{~s}$ lag allows 268 time for cerebral blood flow changes associated with speech to take place.

269 The analysis of the animation task data consists of a standard sequence of processing steps, 270 following work by Deppe, Knecht, Henningsen, and Ringelstein (1997), including removal of the 271 heartbeat (heart cycle integration), signal normalisation, artefact rejection, epoching and baseline 272 correction. Following this initial processing, a Laterality Index (LI) was calculated for each 273 individual. First, grand mean curves for blood flow velocity in each MCA were produced by 274 averaging across all valid trials at each time point during the course of a trial. Then, the time 275 point reflecting the greatest difference in velocity between the two MCAs during the period of 276 interest was identified, and a $2 \mathrm{~s}$ window centred on that point was constructed. The mean 277 velocity in the left MCA during this window minus the mean in the right MCA gave the LI.

278 In previous studies, we have used Matlab to perform this analysis using the DopOSCCI 279 toolbox (Badcock, Holt, Holden, \& Bishop, 2012). However, our research group is now moving 280 to using $\mathrm{R}$ across the whole pipeline of data processing, so we created an $\mathrm{R}$ script to perform the 281 same sequence of operations, while also analysing all files using DopOSCCI for comparison. The 282 R script incorporated one additional feature, which enabled the identification of single data points 283 during trials where there had been very brief signal dropout (typically due to movement of the 284 probe) for substitution by the mean value of that trial. This avoids losing the whole trial because 285 of one aberrant data point, while also correcting for an extreme value that could affect the signal 286 normalisation procedure. Following Groen et al. (2012) we excluded data from children who had 287 fewer than 12 accepted trials, as the LI is likely to be unreliable when based on such a small 288 amount of data.

289 In addition to computing LIs, we also assigned each participant to a laterality category. For 290 this purpose, we tested whether the LI was significantly different from zero, with the $p$-value set 291 to .05. To enable significance-testing, the standard error of each participant's LI was calculated 292 thus: firstly, trial-by-trial LIs were computed for every valid trial during the $2 \mathrm{~s}$ window from 293 which the participant's overall LI was derived, and then the standard deviation of these trial-by294 trial LIs was divided by the square root of the number of valid trials to give the standard error of 295 the overall LI. Next, the standard error was multiplied by \pm 1.96 and added to the LI, giving $95 \%$ 
296 confidence intervals around the LI. If these CIs did not cross with zero, laterality was classified 297 as left or right depending on direction, and bilateral where the CIs did cross zero. Note that there 298 is always a concern that a coding of bilateral laterality could result if data were merely noisy.

299 In addition to overall LI and laterality category, several other measures were taken. Firstly, 300 LIs were derived separately from odd and even trials to allow computation of split half reliability. 301 Secondly, we calculated the difference in blood flow between the mean for the period of interest 302 relative to the mean of the immediately preceding baseline, averaged across all valid trials; this 303 was done separately for the left and right MCAs. Since blood flow velocity is normalized to a 304 mean of 100 and baseline-corrected during signal processing, a positive value indicates a percent 305 increase during the period of interest, and a negative value a percent decrease. Finally, the mean 306 number of words spoken by the child during valid trials was recorded.

307 Left hemisphere dominance. According to the left-brain bias model (Bishop, 2013), if an 308 individual shows consistent left hemisphere dominance across different laterality measures, it is 309 likely to indicate that they belong to the left-bias category. On the other hand, inconsistency 310 across measures would indicate that an individual is of the no-bias category, which may be a risk 311 factor for DLD. To test this notion, children were identified as consistently left dominant if they 312 were categorised as left lateralised for language by fTCD, and if they were categorised as right313 handed on both handedness tasks. In all other cases, children were classed as not having 314 consistent left hemisphere dominance.

\section{Procedure}

316 Ethical approval was obtained for the study in 2011 from the Berkshire NHS Research 317 Ethics Committee (reference 11/SC/0096), and data collection started in August of that year, 318 finishing in October 2016. Where families had expressed interest in the study, they were 319 interviewed by telephone to assess whether the children were likely to meet inclusion criteria, and 320 if so, an appointment was made to see the twins at home or at school, depending on parental 321 preference. Written consent was obtained from a parent/caregiver for their child's participation, 322 and children signed a simplified assent form. Families were widely dispersed around the UK, 323 including Northern Ireland, Scotland, Wales and England, so testing was scheduled where 324 possible to minimise travel. During the course of recruitment, which lasted for a period of five 
325 years, a total of eight research assistants as well as the senior author were involved in assessing 326 children. In some cases, two testers worked together, each seeing one twin, and in others, a single 327 tester saw both children sequentially. The assessment was conducted in a single session lasting 328 between 2-3 hours per child, with breaks where needed.

\section{Data analysis}

330 Study data were analysed using R software (R Core Team, 2016), with the main database 331 managed using REDCap electronic data capture tools hosted at the University of Oxford (Harris 332 et al., 2009). Original data are available on Open Science Framework (https://osf.io/ksqrf/? 333 view_only=54f013aaf65d45a5924748179538756d).

334 Results from the language/cognitive test battery were used to categorise children as having 335 DLD if they scored more than 1 SD below population norms on two or more out of 13 336 language/literacy measures, and as TD if they scored below this threshold on no more than one 337 measure. In previous studies, we have excluded children who met this criterion solely on literacy 338 measures (TOWRE and NARA-II); in the current sample, 10 children fell in this category and 339 were included as DLD, on the basis that prior research has found atypical laterality in adults with 340 dyslexia (Illingworth \& Bishop, 2009). The mean number of tests on which a child with DLD 341 underperformed was $4.21(\mathrm{SD}=2.49)$. It should also be born in mind that the children with DLD 342 had lower nonverbal ability than the TD children. In a multilevel model with performance IQ as 343 dependent variable, group (TD or DLD) as a fixed factor and twin pair membership as a random

344 factor, group showed a significant effect, $t(254.83)=5.68, p<.001$. Marginal mean Performance 345 IQ for the TD children was 106.95 [SD = 14.21] and for the children with DLD was 97.89 [SD = 346 13.81]. Cohen's $d$ of the between-groups difference was 0.65. Notably, the IQ difference in this 347 sample was largely down to the TD children "overperforming"; the mean for the children with 348 DLD is close to the assumed population mean (i.e. 100).

\section{INSERT TABLE 2}

To test our main hypothesis that DLD would be associated with reduced laterality, we made 351 between-groups comparisons of means of quantitative variables or proportions of categorical 352 variables. Table 2 shows the full set of independent and dependent variables considered in the 353 analysis. Since participants were twins, it was necessary to account for the lack of independence 
354 of observations (Kenny, Kashy, \& Cook, 2006), and so we adopted a multilevel modelling

355 approach analogous to that used by Brookman, McDonald, McDonald, and Bishop (2013).

356 To test whether mean fTCD LI differed between the DLD and TD groups, we used a 357 multilevel model that considered group as a fixed effect and twin pair membership as a random 358 effect. This model was run using the lme4 package (Bates, Mächler, Bolker, \& Walker, 2015) to 359 specify the model, with the lmerTest package used for significance testing and generating 360 estimated marginal means (Kuznetsova, Brockhoff, \& Christensen, 2016). We also carried out 361 this same analysis for the mean percent change in blood flow during the period of interest relative 362 to the baseline in the left MCA and in the right MCA separately. A multinomial multilevel 363 model, with categorisation of fTCD laterality as left, bilateral or right as the dependent variable, 364 was used to test whether individuals with DLD were more likely than TD individuals to show 365 right or bilateral compared to left laterality. The model estimated two logit equations, each 366 comparing left laterality to one of the atypical lateralities, and assigned predicted log-odds to 367 each comparison. We used the MCMCglmm package in R to run the model (Hadfield, 2010). As 368 the package adopts a Bayesian approach (Markov Chain Monte-Carlo iterative sampling), 95\% 369 credible intervals were calculated around the predicted log-odds, and we took no overlap with 370 zero to indicate significance. Predicted log odds are reported as odds ratios for ease of 371 interpretation. We also plot the grand average curves for both groups showing change in flow in 372 the two MCAs and change in laterality over the course of a trial.

373 We assessed the ability of group to predict the quantitative handedness measures (the QHP 374 and the adapted EHI) using inflated beta regressions. These were implemented with the 375 GAMLSS package (Stasinopoulos \& Rigby, 2007). For analysis, scores were rescaled to range 376 between 0 and 1 . As the handedness measures are bounded and skewed, they approximate a beta 377 distribution; this can be modelled optimally using a beta regression (Ferrari \& Cribari-Neto, 378 2004). While zeros and ones are not possible within a beta distribution, these can be scored on the 379 handedness measures (i.e. extreme left and extreme right handedness), and therefore we used 380 inflated beta regression, which can incorporate these values through a mixture model (Ospina \& 381 Ferrari, 2012). To our knowledge, existing packages in R do not allow the inclusion of a random 382 effect in inflated beta regression. Therefore, instead of modelling twin pair membership as a 383 random effect, we ran the regressions in two replication samples with twin one in one sample and 
384 twin two in the other, in order to deal with the non-independence of observations. Twins were

385 arbitrarily labelled as such at the start of the study, so these qualify as random samples. All 386 models included the logit function of the handedness measure (either the adapted EHI or QHP) as

387 the dependent variable and group as predictor. Beta coefficients and associated $p$-values are 388 reported for group, and we required $p<.05$ in both samples for a significant effect of group on 389 that particular measure. In an attempt to replicate the previously reported relationship between 390 language disorder and a reduced tendency to reach across the midline (Bishop, 2005; E. L. Hill \& 391 Bishop, 1998), we also assessed whether the probability of reaching to the seven spatial positions 392 in the QHP task differed between the DLD and TD groups. A multilevel model was applied to the 393 data using the lme4 package, with twin pair membership as a random effect, spatial position as a 394 within factor and group as a between factor. Main effects and the interaction are reported.

395 To complete the main analysis, we tested the prediction that individuals with DLD would 396 show less evidence of consistent left hemisphere dominance on the three laterality measures used 397 in this study. An individual was coded as left hemisphere dominant if they were left lateralised on 398 fTCD, and were right-handed on both the adapted EHI and QHP; otherwise, they were coded as 399 not consistently left hemisphere dominant. In testing the hypothesis, we ran a multilevel binary 400 logistic regression using the R package mse4, with left hemisphere dominance as the fixed effect 401 and twin pair membership as a random effect. We report significance testing of the fixed effect 402 and the odds ratio with profile likelihood 95\% CIs that a TD individual would be consistently left 403 hemisphere dominant compared to a DLD individual being consistently left hemisphere 404 dominant.

405 A subsidiary aim was to see whether we could replicate associations between language 406 laterality and language tests found by Groen et al. (2012). Analysis was conducted in two 407 replication samples, as described above, so that data from only one twin of each twin pair 408 contributed to each analysis. Spearman's method was used, since the laterality index is not 409 normally distributed, and all 13 language measures as well as performance IQ were included. We 410 applied the Holm-Bonferroni correction to each of the two sets of correlations to adjust for 411 multiple testing, and required a correlation to be significant in both samples for it to be classed as 412 a true effect. 


\section{Results}

\section{Preliminary Analysis of fTCD language laterality}

415

416

417

418

419

420

421

422

423

424

425

426

427

428

429

430

431

432

433

434

435

436

437

438

439

440

441

Table 3 shows descriptive statistics for all measures; the language measures are reported as standard scores. In our sample, fTCD results indicated that $61.50 \%$ of the typically developing children were left lateralised for language in the animation description task; $18.60 \%$ were categorised as bilateral and $19.90 \%$ as right lateralised. Respective percentages for the children with DLD were $72.90 \%$ as left, $17.80 \%$ as bilateral and $9.30 \%$ as right.

\section{INSERT TABLE 3}

We checked the reliability of the fTCD LI by computing the correlation between the LIs calculated separately for even and odd trials. Split-half reliability was excellent at the full sample level, $r=.84$, and when dividing participants into the TD children, $r=.86$, and those with DLD, $r=.78$. Number of words spoken during valid trials did not predict laterality index, $r=.03, p=$. 614 , indicating that any differences in laterality detected by fTCD cannot be attributed to quantity of speech produced. This impression is supported by a strikingly similar mean number of words produced by left-lateralised children $(M=20.06, S D=4.86)$ compared to bilateral $(M=19.43$, $\mathrm{SD}=5.38)$ and right-lateralised children $(\mathrm{M}=19.87, \mathrm{SD}=5.38)$. We also checked whether the two groups differed in terms of the number of fTCD trials included in analysis. There was a significant difference, $t(203.37)=2.62, p=.009$, with more trials available for the TD children $(\mathrm{M}=26.78, \mathrm{SD}=3.56)$ than the children with $\mathrm{DLD}(\mathrm{M}=25.48, \mathrm{SD}=4.18)$. The effect size was small (Cohen's $d=0.33$ ). Finally, we checked for any sex and age differences in LI using a multilevel model with twin pair membership as a random effect and sex and age as fixed effects. Age showed no effect, $t(147.58)=0.50, p=.617$, but sex did, $t(198.45)=-2.24, p=.026$. Marginal mean LI for boys was 2.10 [SD = 2.78] and for girls was 1.32 [SD $=2.78]$. The effect size was small (Cohen's $d=0.28$ ). As we did not make any prediction about sex in this study, we did not include the effect of sex in our hypothesis-testing models. However, seeing as it showed a relationship with fTCD laterality, we do report some exploratory analysis of sex by group interactions in Supplemental Information. 
442 (Newman, 1928), hormonal transfer (Elkadi, Nicholls, \& Clode, 1999) and birth order (James \& 443 Orlebeke, 2002), and due also to an increased general risk of perinatal complications. Early 444 adversity affecting the left hemisphere may be associated with the compensatory development of 445 atypical right hemisphere dominance, promoting "pathological left-handedness" (Annett, 1985), 446 although this theory was not supported in a large longitudinal data-set that found no relationship 447 between atypical handedness and birth stress (McManus, 1981). Medland et al. (2009) presented 448 evidence from 54, 270 twins and their siblings for an absence of any twin-specific effects: 449 prevalence of left-handedness did not differ between twins by zygosity, sex or birth order, and 450 nor did it differ between twins and their non-twin siblings.

451 While the empirical findings speak against twin-specific effects, we nonetheless considered 452 whether laterality was unusual in the typically developing twins compared to single-born 453 children, before proceeding to the main analysis. We compared the TD children in this sample to 454 a previous sample of single-born children $(\mathrm{N}=58)$ tested using the same task with fTCD (Groen 455 et al., 2012). Although more of the TD twins show right-sided laterality than the children in the 456 previous sample, overlapping 95\% high density intervals between the two groups indicate no 457 difference in central tendency. See the pirate plot below in Fig. 2 for the distributions of LIs. 458 Handedness, as measured by the adapted EHI was also very similar among the TD twins and the single-born sample [twins, $\mathrm{M}=65.67, \mathrm{SD}=56.37$; single-born children, $\mathrm{M}=63.57, \mathrm{SD}=44.53$ ].

The twin-specific effects outlined above are predicted to influence laterality based on zygosity. Mirror-imaging, for instance, is thought to increase the risk of atypical laterality in MZ 462 twins if the embryo does not split until after the left-right axis forms (Newman, 1928). In testing 463 for differences in zygosity in a multilevel model with twin pair membership as a random effect, 464 zygosity was not significant when included as a fixed effect, $t(137.14)=0.82, p=.414$. Marginal 465 means [95\% CIs] were 1.83 [1.32 - 2.34] for MZ twins, 1.59 [1.01 - 2.18] for DZ same sex twins and 1.45 [0.68 - 2.23] for DZ twins. From this analysis, we suggest that twinning is not related to atypical laterality. 
469

470

471

472 473

474 475

476

477

478

479

480

481

482

483

484

485

486

487

488

489

490

491

492

493

494

495

\section{Main Analysis}

The first step of analysis involved testing whether the laterality measures predicted whether or not a child was diagnosed with DLD using multilevel modelling. In the first model, the fTCD laterality index was used as a continuous measure, and in the second, a categoric measure was used, with children grouped as left, bilateral or right lateralised based on whether confidence intervals for the LI crossed with zero. Since we had clear a priori expectations that any group differences would involve reduced laterality in the DLD group, we did not correct for multiple testing. Contrary to hypothesis, there was no between-groups difference in fTCD LI, with the group factor being non-significant, $t(242.63)=1.32, p=.190$. See Table 4 for marginal means and associated 95\% CIs.

Following Whitehouse and Bishop (2008), we also assessed via multilevel modelling whether mean blood flow in the left and right MCAs during the period of interest relative to the mean of the baseline was significantly different in the two groups. As can be seen in the grand average plots shown in Fig. 3, the time course of the changes in blood flow was very similar in both groups. Blood flow peaks bilaterally at the start of the trial, and it is only when flow returns to baseline levels in both MCAs that the left-sided bias emerges, with flow in the right MCA dropping below that in the left MCA.

\section{INSERT FIGURE 3}

There was no effect of group (TD or DLD) on mean percent change in flow in the left MCA, $t(258.28)=1.94, p=.053$, or the right MCA, $t(260.97)=1.72, p=.086$, during the period of interest. See Table 4 for marginal means and associated 95\% CIs. The non-significant trend for slightly lower flow in the TD group was likely driven by a greater range in blood flow in the talk phase relative to the baseline for the TD children [left: -15.80 to $+9.00 \%$; right: -18.40 to $+10.00 \%$ ], compared to those with DLD [left: -9.80 to $+9.00 \%$; right: -8.90 to $+6.00 \%$ ] Nonetheless, the overall impression of this analysis is of no significant between-group differences in cerebral blood flow during the language task. 
Next, we specified a multinomial model testing for categoric differences in laterality in

497 those with and without DLD. There was an effect of group on right compared to left laterality, $498 p \mathrm{MCMC}=.007$, although this went in the opposite direction to that hypothesised, with an over-

499 representation of TD children showing right lateralised language. There was no effect of group on 500 bilateral compared to left laterality, $p \mathrm{MCMC}=.503$. The predicted odds ratio [95\% credibility

501 intervals] of a TD child compared to a child with DLD being right rather than left lateralised was 5023.42 [1.20, 8.90], and for being bilateral rather than left lateralised was 1.33 [0.57, 2.85].

503 We then moved to look at handedness, testing the hypothesis that the DLD group was less 504 right-handed than the TD children. We ran inflated beta regression models with the adapted EHI 505 and QHP as dependent variable, running each model in two replication samples, with random 506 allocation of one twin to one sample and the other twin to the other. With logit-transformed 507 handedness scores on the adapted EHI as dependent variable, the coefficients for group were non508 significant in sample $1, \quad \beta=.02, t(131)=.08, p=.939$, and sample $2, \quad \beta=-.07, t(132)=$ $509-.31, p=.754$. This was the same for the regressions predicting logit-transformed QHP scores in 510 sample 1, $\quad \beta=.33, t(131)=1.38, p=.171$, and sample $2, \quad \beta=-.24, t(132)=-.31, p=.284$.

511 Note that sex and age showed no relationship with handedness, so these were not incorporated in 512 any models.

513 We also tested the hypothesis that children with DLD were less likely in the QHP task to 514 reach across the midline with the right hand to a spatial position on the left side of the body, 515 indicating weaker hand preference, as previously reported (Bishop, 2005; E. L. Hill \& Bishop, 516 1998). A multilevel model was run with twin pair membership as a random effect, group as a 517 between factor and spatial position as a within factor. There was a large main effect of spatial 518 position, $t(1,687.20)=12.74, p<.001$, with individuals being less likely to reach across the 519 midline with the right hand to a spatial position on the left. However, there was no main effect of 520 group, $t(1,836.70)=1.61, p=.108$, and contrary to previous reports, no interaction between 521 DLD status and spatial position, $t(1,687.20)=0.89, p=.376$, indicating no between-groups 522 differences in strength of hand preference. See Fig. 4 for a plot of the probability of right hand 523 reaches to each spatial location by group. 
525 For the last part of the main analysis, we evaluated the hypothesis that inconsistency of left 526 hemisphere dominance was associated with DLD. Firstly, the TD and DLD groups were divided 527 into subgroups based on the combination of tasks on which a child showed evidence of left 528 hemisphere dominance; see Table 5 for the number of children falling into each group.

Then we tested whether there was a between-groups difference in the number of children 531 falling into the 1,1,1 category versus any other category. For this purpose, we used a multilevel 532 logistic regression, with group (TD or DLD) as a fixed effect and twin pair membership as a 533 random effect. DLD showed no relationship with consistency of left hemisphere dominance, $z=$ $5341.48, p=.139$, with this trait as frequent in the DLD group as in the TD children. The predicted 535 odds [95\% CIs] for a child with DLD compared to a TD child showing consistent left hemisphere 536 dominance was $1.48[0.89,2.52]$.

537 Since the overall percentage of children showing consistent left hemisphere dominance on 538 the laterality measures was only around 50\%, we checked whether relationships existed between 539 the variables at the sample level, as lack of a relationship would complicate interpretation of that 540 preceding analysis. For this purpose, we used multilevel models, with twin pair membership as a 541 random effect. In the first model, we verified that the handedness measures were related. As 542 expected, handedness measured by the adapted EHI was a significant predictor of quantitative 543 handedness $(\mathrm{QHP}), p<.001$. The measures shared a moderate amount of variance over and

544 above the effect of twin pair membership, pseudo $R^{2}=.21$. On the other hand, in two similar 545 multilevel models with fTCD LI as dependent variable and one handedness measure as a fixed 546 effect in each, the adapted EHI was not a significant predictor of LI, $p=.878$, and nor was the 547 QHP, $p=.893$. Therefore, manual and language laterality were not related in our sample, though 548 the separate measures of manual laterality were. With this in mind, we checked whether 549 individual departures from the group pattern of right-handedness were a predictor of DLD in a 550 second multilevel logistic regression. An individual was coded as right-handed (1) if they were 551 right-handed on both measures, and coded as 0 otherwise. The fixed effect of group (TD or DLD) 552 was also non-significant in this model, $z=.86, p=.388$. The predicted odds [95\% CIs] for a child 553 with DLD compared to a TD child showing consistent right-handedness was 1.28 [0.73, 2.29]. 
554 In the second step of analysis, we tested the relationship between language and laterality

555 from the opposite direction, using language measures as predictors, aiming to replicate the effects

556 reported by Groen et al. (2012). We computed Spearman's correlations between each language

557 measure and the fTCD laterality index in the two replication samples. However, no uncorrected

558 correlation replicated across samples, and no correlation within a sample survived a Holm-

559 Bonferroni correction. This was also the case when correlations were computed using absolute

560 laterality indices, i.e. when testing the relationship between the strength of laterality rather than

561 the laterality index per se, which is a combined measure of strength and direction.

562 Some of the children recruited into the study (though excluded in the main analysis) were 563 reported by their parent(s) as having autism $(n=12)$. For completeness of reporting, we provide 564 fTCD laterality results for this group below. Useable fTCD data were collected from 10 children 565 with ASD, all of whom had an IQ above $70(\mathrm{M}=100.18, \mathrm{SD}=12.69)$. These children showed

566 relatively low left lateralisation for language function when assessed using fTCD, both in terms 567 of the quantitative $\mathrm{LI}(\mathrm{M}=1.18, \mathrm{SD}=3.03)$, and in terms of categoric laterality (five were left 568 lateralised, two showed bilateral language, and three were right lateralised). Two of the children

569 were girls, both of whom were left lateralised. This analysis should not be given undue weight 570 given the small sample, and it should be borne in mind that autism diagnoses were reported by 571 parents, and were not confirmed in the course of this study using standardized clinical 572 instruments.

\section{Discussion}

574

The present study evaluated whether reduced laterality for language was more common

575 among those with developmental language problems. In our sample of 263 twins, we did not find 576 any evidence for increased prevalence of atypical laterality in those with developmental language 577 disorder. On the contrary, the fact that so many of the typically developing children in the sample 578 were right lateralised for language when assessed using fTCD (19.90\%) indicates that atypical

579 laterality is not inconsistent with the development of typical language skills.

580 This study filled the need expressed by a recent review of the neuroimaging literature on 581 childhood language disorder (Mayes et al., 2015) for a large confirmatory study of whether 582 patterns of cerebral lateralisation are disrupted in language disorder. Mayes et al. (2015) indicated 
583 that several exploratory studies provided limited evidence of neural differences, including

584 laterality differences, in samples of language-impaired children, however methodological

585 heterogeneity and variability in language phenotyping made it difficult to evaluate the strength of

586 the evidence. The small sample sizes (no study included in the review exceeded 36 participants)

587 and possible publication bias are also obvious problems thought these went unmentioned by the

588 reviewers. Based on the large sample size of our present study, we suggest that previous reports

589 of reduced left hemispheric dominance for language among those with language problems (e.g.

590 Illingworth \& Bishop, 2009; Whitehouse \& Bishop, 2008) are likely to have been false positives.

591 This result speaks to the chronic problem of low power that afflicts the neuroscience literature;

592 Button et al. (2013) calculated a median statistical power of only $21 \%$ across 730 neuroscience

593 studies incorporated in 48 meta-analyses published in 2011. Low powered studies are likely to

594 fail to detect true effects where they exist, and the lower the average power of a study, the more

595 the research literature will become disproportionately represented by false positives (Sterne \&

596 Smith, 2001). This is because, as power and therefore the number of true positives reduce, the

597 number of false positives remains constant, since this is set by the $p$-value threshold. Low power

598 should therefore generate low confidence that a positive finding in the research literature is a real

599 effect, and this is before factoring in any further distortions created by publication bias and

600 "flexible" analytical and/or reporting practices (Button et al., 2013). The moral of the present

601 study is that the effects reported by small underpowered neuroscience studies should not be

602 trusted until they are replicated in large well-powered studies.

603 The lack of a relationship between handedness and DLD in the present study also calls into 604 question the mixed literature surrounding motor laterality and neurodevelopmental disorders. In 605 particular, our failure to replicate a between-groups difference in the probability of reaching 606 across the midline with the right hand indicates that this may not be a marker of compromised 607 neurodevelopment relevant to language disorder, as previously suggested (Bishop, 2005; E. L. 608 Hill \& Bishop, 1998). We also failed to support the prediction of the left-brain bias model 609 (Bishop, 2013) that reduced evidence of left hemisphere dominance would be found in the DLD 610 group. This was the case even though around $50 \%$ of the children did not show evidence of left 611 hemisphere dominance on all three laterality variables. Indeed, there was a non-significant trend 612 in the opposite direction hypothesised, with the TD group showing slightly less left hemisphere 613 dominance across measures. 
614 While the hypothesis was not supported, it may be premature to reject a possible role for a 615 lack of consistent left hemisphere dominance in the development of language problems. Since 616 this study used handedness measures and only one index of language lateralisation, we were not

617 able to test a key prediction of the left-brain bias model, which stresses the importance of 618 consistent left lateralisation across different language functions. By contrast, our assessment of 619 left-hemisphere dominance was based on one language laterality measure and two measures of 620 handedness. The relationship between handedness and language laterality measured by

621 fTCD/fMRI is indirect at best (Badzakova-Trajkov, Häberling, Roberts, \& Corballis, 2010;

622 Groen et al., 2013; Mazoyer et al., 2014; M. Somers et al., 2015), and in this respect, it is notable 623 that the present study found that neither handedness measure predicted fTCD LI. This indicates 624 that cerebral dominance for motor and language functions is likely to develop by largely

625 independent processes, meaning that inconsistency between handedness and language laterality at 626 the individual level need not reflect problems with hemispheric specialization. For a stronger test 627 of the left-brain bias model, it would be necessary to identify individuals who do not show 628 consistent left laterality across language tasks, which evoke moderately correlated patterns of 629 lateralisation at the group level. In future work, we plan to test the prediction of the model that 630 inconsistent lateralisation across language tasks will be associated with greater risk for DLD.

631 Nevertheless, if the left-brain bias account were valid, we would expect to see at least a trend for 632 reduced language lateralisation in the DLD group on the one measure we did have. The failure to 633 observe such a trend in this large sample does weaken support for the model.

634 The absence of any relationship between lateralisation and language development cannot be 635 attributed to a lack of individual variability in the data-set. On the contrary, considerable 636 individual differences were evident in our sample - especially among the typically developing 637 children, of whom nearly a fifth were right-lateralised during the animation description task used 638 in our study. This finding remains in need of explanation. As one of our reviewers suggested, a 639 possibility is that different children used different strategies to complete the animation 640 description task. While it is wholly speculative, some children may have tended to construct 641 mental images from the videos when describing them, which may have placed more demand on 642 visuospatial cognition, which is typically right lateralised (Badzakova-Trajkov, Häberling, 643 Roberts, \& Corballis, 2015; Jansen et al., 2004). 
644 There is a possibility that the null effect reported in this study is due to fTCD lacking the

645 spatial resolution to pick up between-group differences if these are very fine-grained and focally

646 located in the brain. All the same, if the distribution of language representation across the frontal

647 and temporoparietal regions supplied by the middle cerebral artery does show widespread

648 differences in laterality in DLD, we would expect to see the effects using fTCD. fTCD is highly

649 sensitive to language-related activity, as confirmed by the high correlation reported between

650 laterality indices produced using fTCD and fMRI for language tasks (M. Deppe et al., 2000; M.

651 Somers et al., 2011). Furthermore, fTCD consistently shows a similar level of sensitivity to fMRI

652 for the other commonly studied lateralised cognitive process, visuospatial function (Hattemer et

653 al., 2011; Jansen et al., 2004), and it also identifies categoric language dominance at a high level

654 of agreement with the Wada test, in which the direct effect of anaesthetic injected into each MCA

655 is observed on speech during neurosurgery (Knake et al., 2003; Knecht et al., 1998). We can

656 therefore be confident in fTCD as a valid tool for measuring language lateralisation. We can also

657 trust that the LIs reported in the present study reflected stable cerebral responses on a trial-by-

658 trial basis given the high split-half reliability. Indeed, reproducibility of "gold-standard" fMRI

659 measurements (Adcock, Wise, Oxbury, Oxbury, \& Matthews, 2003; Fernández et al., 2003;

660 Jansen et al., 2006; Wilson, Bautista, Yen, Lauderdale, \& Eriksson, 2017) is often lower than

661 what is typically found for the fTCD LI (e.g. Bishop et al., 2009).

\section{Conclusions}

663 In a large sample of twins oversampled for language problems, the present study failed to

664 find evidence for atypical laterality, either in terms of handedness or cerebral lateralisation for

665 language, in those with DLD. Theories have proposed that disruption to the typical left

666 hemisphere dominance for language may be a neurobiological correlate of language problems

667 (e.g. Annett, 2002; Bishop, 2013; T. J. Crow et al., 1998), and empirical studies of very small

668 samples have supported that view (Illingworth \& Bishop, 2009; Whitehouse \& Bishop, 2008).

669 However, the present study did not replicate these findings, and we suggest that they are likely to

670 have been false positives. In our large twin sample, fTCD testing revealed substantial individual

671 variation in laterality, but the bias for left brain dominance for language showed no difference at

672 the group level between those with and without DLD. We conclude, therefore, that reduced left

673 hemisphere dominance is unlikely to be implicated in language disorder. 
675 We offer warmest thanks to the families who took part in the study, and school staff who 676 helped facilitate assessment arrangements. The study would not have been possible without the 677 hard work and dedication of a series of research assistants who conducted the assessments, often 678 travelling all over the UK to do so: Eleanor Payne, Nicola Gratton, Georgina Holt, Annie 679 Brookman, Elaine Gray, Louise Atkins, Holly Thornton and Sarah Morris. We also thank Paul A. 680 Thompson for expert advice on statistical analysis and Margriet Groen for making data available 681 for comparison with a previous study.

\section{References}

683

684

685

686

687 688 Erlbaum.

689 690 Press.

691

692 693

694 695 696 697

Annett, M. (2002). Handedness and brain asymmetry. Hove, East Sussex: Psychology

Adcock, J. E., Wise, R. G., Oxbury, J. M., Oxbury, S. M., \& Matthews, P. M. (2003).

Quantitative fMRI assessment of the differences in lateralization of language-related brain activation in patients with temporal lobe epilepsy. NeuroImage, 18(2), 423-438. doi:https://doi.org/10.1016/S1053-8119(02)00013-7

Annett, M. (1985). Left, right, hand and brain: The right shift theory. London, England:

Badcock, N. A., Bishop, D. V. M., Hardiman, M. J., Barry, J. G, \& Watkins, K. E. (2012). Co-localisation of abnormal brain structure and function in specific language impairment. Brain \& Language, 120, 310-320. doi:htpps://doi.org/10.1016/j.bandl.2011.10.006

Badcock, N. A., Holt, G., Holden, A., Bishop, D. V. M. (2012). dopOSCCI: A functional transcranial Doppler ultrasonography summary suite for the assessment of cerebral lateralization of cognitive function. Journal of Neuroscience Methods, 204(2), 383-388. doi:https://doi.org/10.1016/i.jneumeth.2011.11.018

PeerJ reviewing PDF | (2017:10:21150:1:0:ACCEPTED 8 Dec 2017) 
698 Badzakova-Trajkov, G., Häberling, I. S., \& Corballis, M. C. (2010). Cerebral asymmetries 699 in monozygotic twins: an fMRI study. Neuropsychologia, 48(10), 3086-3093.

700 doi:https://doi.org/10.1016/j.neuropsychologia.2010.06.020

701 Badzakova-Trajkov, G., Häberling, I. S., Roberts, R. P., \& Corballis, M. C. (2010).

702 Cerebral asymmetries: Complementary and independent processes. PLOS ONE, 5(3), e9682.

703 doi:https://doi.org/10.1371/journal.pone.0009682

704 Bates, D., Mächler, M., Bolker, B., \& Walker, S. (2015). Fitting linear mixed-effects

705 models using \{lme4\}. Journal of Statistical Software, 67(1), 1-46.

706 doi:https://doi.org/10.18637/jss.v067.i01

707

708

709

710

711

712

713

714

715

716

717

718

719

720

721

722

Berl, M. M., Mayo, J., Parks, E. N., Rosenberger, L. R., VanMeter, J., Ratner, N. B., ... Gaillard, W. D. (2014). Regional differences in the developmental trajectory of lateralization of the language network. Human Brain Mapping, 35(1), 270-284.

doi:https://doi.org/10.1002/hbm.22179

Bernal, B., \& Altman, N. R. (2003). Speech delay in children: A functional MR imaging study. Radiology, 229(3), 651-658. doi:https://doi.org/10.1148/radiol.2293021746

Bishop, D. V. M. (1990). How to increase your chances of obtaining a significant association between handedness and disorder. Journal of Clinical and Experimental Neuropsychology, 12(5), 812-816. doi:https://doi.org/10.1080/01688639008401022

Bishop, D. V. M. (2001). Individual differences in handedness and specific speech and language impairment: evidence against a genetic link. Behavior Genetics, 31(4), 339-51. doi:https://doi.org/10.1023/A:1012239617367

Bishop, D. V. M. (2003). The children's communication checklist version 2 (CCC-2). London, England: Psychological Corporation.

Bishop, D. V. M. (2005). Handedness and specific language impairment: A study of 6-yearold twins. Developmental Psychobiology, 46(4), 362-369. doi:https://doi.org/10.1002/dev.20062 
723 Bishop, D. V. M. (2013). Cerebral asymmetry and language development: Cause, correlate, 724 or consequence? Science, 340(6138). doi:https://doi.org/10.1126/science.1230531

725 Bishop, D. V. M., Badcock, N. A., \& Holt, G. (2010). Assessment of cerebral lateralization 726 in children using functional transcranial Doppler ultrasound (fTCD). Journal of Visualized 727 Experiments: JoVE, 43. doi:https://doi.org/10.3791/2161

728 Bishop, D. V. M., Holt, G., Whitehouse, A. J. O., \& Groen, M. (2014). No population bias 729 to left-hemisphere language in 4-year-olds with language impairment. PeerJ, 2, e507. 730 doi:https://doi.org/10.7717/peerj.507

731 Bishop, D. V. M., Ross, V. A., Daniels, M. S., \& Bright, P. (1996). The measurement of 732 hand preference: A validation study comparing three groups of right-handers. British Journal of 733 Psychology, 87(2), 269-285. doi:https://doi.org/10.1111/j.2044-8295.1996.tb02590.x

734 Bishop, D. V. M., Watt, H., \& Papadatou-Pastou, M. (2009). An efficient and reliable 735 method for measuring cerebral lateralization during speech with functional transcranial Doppler 736 ultrasound. Neuropsychologia, 47(2), 587-590.

737 doi:https://doi.org/10.1016/j.neuropsychologia.2008.09.013

738 Brookman, A., McDonald, S., McDonald, D., \& Bishop, D. V. M. (2013). Fine motor 739 deficits in reading disability and language impairment: Same or different? PeerJ, 1, e217. 740 doi:https://doi.org/10.7717/peerj.217

741 Button, K. S., Ioannidis, J. P. A., Mokrysz, C., Nosek, B. A., Flint, J., Robinson, E. S. J., \& 742 Munafo, M. R. (2013). Power failure: why small sample size undermines the reliability of 743 neuroscience. Nature Reviews Neuroscience, 14, 365-376. doi:https://doi.org/10.1038/nrn3475

744 Crow, T. J., Crow, L. R., Done, D. J., \& Leask, S. (1998). Relative hand skill predicts 745 academic ability: Global deficits at the point of hemispheric indecision. Neuropsychologia, 746 36(12), 1275-1282. doi:https://doi.org/10.1016/S0028-3932(98)00039-6

747 de Guibert, C., Maumet, C., Jannin, P., Ferré, J.-C., Tréguier, C., Barillot, C., ... Biraben, 748 A. (2011). Abnormal functional lateralization and activity of language brain areas in typical 
749 specific language impairment (developmental dysphasia). Brain, 134(10), 3044-3058.

750 doi:https://doi.org/10.1093/brain/awr141

751 Deppe, M., Knecht, S., Henningsen, H., \& Ringelstein, E. B. (1997). AVERAGE: a

752 Windows program for automated analysis of event related cerebral blood flow. Journal of

753 Neuroscience Methods, 75(2), 147-154. doi:https://doi.org/10.1016/S0165-0270(97)00067-8

754 Deppe, M., Knecht, S., Papke, K., Lohmann, H., Fleischer, H., Heindel, W., ...

755 Henningsen, H. (2000). Assessment of hemispheric language lateralization: A comparison

756 between fMRI and fTCD. Journal of Cerebral Blood Flow \& Metabolism, 20(2), 263-268.

757 doi:https://doi.org/10.1097/00004647-200002000-00006

758 Eglinton, E., \& Annett, M. (1994). Handedness and dyslexia: A meta-analysis. Perceptual 759 and Motor Skills, 79(3), 1611-1616. doi:https://doi.org/10.2466/pms.1994.79.3f.1611

760 Elkadi, S., Nicholls, M., E., \& Clode, D. (1999). Handedness in opposite and same-sex

761 dizygotic twins: testing the testosterone hypothesis. Neuroreport, 10(2), 333-336.

762 doi:https://doi.org/10.1097/00001756-199902050-00023

763 Ettinger-Veenstra, H. M. van, Ragnehed, M., Hällgren, M., Karlsson, T., Landtblom, A.-

764 M., Lundberg, P., \& Engström, M. (2010). Right-hemispheric brain activation correlates to

765 language performance. NeuroImage, 49(4), 3481-3488.

766 doi:https://doi.org/10.1016/j.neuroimage.2009.10.041

767 Everts, R., Lidzba, K., Wilke, M., Kiefer, C., Mordasini, M., Schroth, G., ... Steinlin, M. 768 (2009). Strengthening of laterality of verbal and visuospatial functions during childhood and 769 adolescence. Human Brain Mapping, 30(2), 473-483. doi:https://doi.org/10.1002/hbm.20523

770 Fernández, G., Specht, K., Weis, S., Tendolkar, I., Reuber, M., Fell, J., ... Elger, C. E. 771 (2003). Intrasubject reproducibility of presurgical language lateralization and mapping using 772 fMRI. Neurology, 60, 969-975. doi:https://doi.org/10.1212/01.WNL.0000049934.34209.2E

773 Ferrari, S. L. P., \& Cribari-Neto, F. (2004). Beta regression for modelling rates and 774 proportions. Journal of Applied Statistics, 31(7), 799-815.

775 doi:https://doi.org/10.1080/0266476042000214501 
776 Frederickson, N., Frith, U., \& Reason, R. (1997). Phonological assessment battery (PhAB). 777 Windsor, England: NFER-Nelson.

778 Friederici, A. D. (2011). The brain basis of language processing: From structure to 779 function. Physiological Reviews, 91(4), 1357-1392.

780 doi:https://doi.org/10.1152/physrev.00006.2011

781 Groen, M., Whitehouse, A. J. O., Badcock, N. A., \& Bishop, D. V. (2012). Does cerebral 782 lateralization develop? A study using functional transcranial Doppler ultrasound assessing 783 lateralization for language production and visuospatial memory. Brain and Behavior, 2(3), 256784 269. doi:https://doi.org/10.1002/brb3.56 between handedness and cerebral lateralisation for language: A comparison of three measures in children. PLOS ONE, 8(5), e64876. doi:https://doi.org/10.1371/journal.pone.0064876 models: The MCMCglmm R package. JSS Journal of Statistical Software, 33(2), 1-22. Informatics, 42(2), 377-381. doi:https://doi.org/10.1016/j.jbi.2008.08.010 (2011). Determination of hemispheric dominance with mental rotation using functional transcranial Doppler sonography and fMRI. Journal of Neuroimaging, 21(1), 16-23.

797 doi:https://doi.org/10.1111/j.1552-6569.2009.00402.x specific language impairment and developmental co-ordination disorder. Laterality: Asymmetries of Body, Brain and Cognition, 3(4), 295-310. doi:https://doi.org/10.1080/713754314 
803 Journal of Neuroscience, 36(16), 4522-4533. doi:https://doi.org/10.1523/JNEUROSCI.3850-

$804 \underline{14.2016}$

805 Illingworth, S., \& Bishop, D. V. M. (2009). Atypical cerebral lateralisation in adults with 806 compensated developmental dyslexia demonstrated using functional transcranial Doppler 807 ultrasound. Brain and Language, 111(1), 61-65. doi:https://doi.org/10.1016/j.bandl.2009.05.002

808 James, W. H., \& Orlebeke, J. F. (2002). Determinants of handedness in twins. Laterality, 7, 809 301-307. Doi:https://doi.org/10.1080/13576500143000320

810 Jansen, A., Flöel, A., Deppe, M., Randenborgh, J. van, Dräger, B., Kanowski, M., \& 811 Knecht, S. (2004). Determining the hemispheric dominance of spatial attention: A comparison 812 between fTCD and fMRI. Human Brain Mapping, 23(3), 168-180.

813 doi:https://doi.org/10.1002/hbm.20055

814 Jansen, A., Menke, R., Sommer, J., Förster, A. F., Bruchmann, S., Hempleman, J., ... 815 Knecht, S. (2006). The assessment of hemispheric lateralization in functional MRI: Robustness 816 and reproducibility. NeuroImage, 33(1), 204-217.

817 doi:https://doi.org/10.1016/j.neuroimage.2006.06.019

818 Kenny, D. A., Kashy, D., \& Cook, W. L. (2006). Dyadic data analysis. New York, NY: 819 Cambridge University Press.

820 Knake, S., Haag, A., Hamer, H. M., Dittmer, C., Bien, S., Oertel, W. H., \& Rosenow, F. 821 (2003). Language lateralization in patients with temporal lobe epilepsy: A comparison of 822 functional transcranial Doppler sonography and the Wada test. NeuroImage, 19(3), 1228-1232. 823 doi:https://doi.org/10.1016/s1053-8119(03)00174-5

824 Knecht, S., Deppe, M., Ebner, A., Henningsen, H., Huber, T., Jokeit, H., \& Ringelstein, E.825 B. (1998). Noninvasive determination of language lateralization by functional transcranial 826 Doppler sonography. Stroke, 29(1), 82-86. doi:https://doi.org/10.1161/01.STR.29.1.82

827 Knecht, S., Dräger, B., Deppe, M., Bobe, L., Lohmann, H., Flöel, A., ... Henningsen, H. 828 (2000). Handedness and hemispheric language dominance in healthy humans. Brain, 123(12), 829 2512-2518. doi:https://doi.org/10.1093/brain/123.12.2512 
830 Korkman, M., Kirk, U., \& Kemp, S. I. (1998). NEPSY: A developmental

831 neuropsychological assessment. San Antonio, TX: Psychological Corporation.

832 Kuznetsova, A., Brockhoff, P., \& Christensen, R. (2016). lmerTest: Tests in linear mixed

833 effects models. R Package Version, 3.0.0. Retrieved from https://cran.r-

834 project.org/package=lmerTest

835 Levinson, H. N. (1988). The cerebellar-vestibular basis of learning disabilities in children, 836 adolescents and adults: Hypothesis and study. Perceptual and Motor Skills, 67(3), 983-1006. 837 doi:https://doi.org/10.2466/pms.1988.67.3.983

838 Lindell, A. K., \& Hudry, K. (2013). Atypicalities in cortical structure, handedness, and 839 functional lateralization for language in autism spectrum disorders. Neuropsychology Review, 840 23(3), 257-270. doi:https://doi.org/10.1007/s11065-013-9234-5

841 Mayes, A. K., Reilly, S., \& Morgan, A. T. (2015). Neural correlates of childhood language 842 disorder: a systematic review. Developmental Medicine and Child Neurology, 57(8), 706-717. 843 doi:https://doi.org/10.1111/dmcn.12714

844 Mazoyer, B., Zago, L., Jobard, G., Crivello, F., Joliot, M., Perchey, G., ... Tzourio845 Mazoyer, N. (2014). Gaussian mixture modeling of hemispheric lateralization for language in a 846 large sample of healthy individuals balanced for handedness. PLOS ONE, 9(6), e101165. 847 doi:https://doi.org/10.1371/journal.pone.0101165

848 McGlone, J. (1980). Sex differences in human brain asymmetry: a critical survey.

849 Behavioral and Brain Sciences, 3(2), 215-227. doi:https://doi.org/10.1017/S0140525X00004398

850 McManus, I. C. (1985). Handedness, language dominance and aphasia: a genetic model.

851 Cambridge University Press: Psychological Medicine Monograph Supplement, 8, 3-40.

852 McManus, I. C. (1981). Handedness and birth stress. Psychological Medicine, 11(3), 485853496.

854 Medland, S. E., Duffy, D. L., Wright, M. J., Geffen, G. M., Hay, D. A., Levy, F., ... 855 Boomsma, D. I. (2009). Genetic influences on handedness: data from 25, 732 Australian and 
856 Dutch twin families. Neuropsychologia, 47(2), 330-337.

857 doi:https://doi.org/10.1016/j.neuropsychologia.2008.09.005

858 Mellet, E., Zago, L., Jobard, G., Crivello, F., Petit, L., Joliot, M., ... Tzourio-Mazoyer, N.

859 (2014). Weak language lateralization affects both verbal and spatial skills: An fMRI study in 297

860 subjects. Neuropsychologia, 65, 56-62.

861 doi:https://doi.org/10.1016/j.neuropsychologia.2014.10.010

862 Neale, M. D. (1997). Neale analysis of reading ability (2nd edn.). Windsor, England:

863 NFER-Nelson.

864 Newman, H. H. (1928). Studies of human twins: II. Asymmetry reversal, of mirror imaging 865 in identical twins. The Biological Bulletin, 55(4), 298-315. doi:https://doi.org/10.2307/1537082

866 Ocklenburg, S., Beste, C., Arning, L., Peterburs, J., \& Güntürkün, O. (2014). The 867 ontogenesis of language lateralization and its relation to handedness. Neuroscience and

868 Biobehavioral Reviews, 43, 191-198. doi:https://doi.org/10.1016/j.neubiorev.2014.04.008

869 Oldfield, R. C. (1971). The assessment and analysis of handedness: The Edinburgh 870 inventory. Neuropsychologia, 9(1), 97-113. doi:https://doi.org/10.1016/0028-3932(71)90067-4

871 Ospina, R., \& Ferrari, S. L. P. (2012). A general class of zero-or-one inflated beta 872 regression models. Computational Statistics \& Data Analysis, 56(6), 1609-1623.

873 doi:https://doi.org/10.1016/j.csda.2011.10.005

874 Piervincenzi, C., Petrilli, A., Marini, A., Caulo, M., Committeri, G., \& Sestieri, C. (2016). 875 Multimodal assessment of hemispheric lateralization for language and its relevance for behavior. 876 NeuroImage, 142, 351-370. doi:https://doi.org/10.1016/j.neuroimage.2016.08.018

877 R Core Team. (2016). R: A language and environment for statistical computing. Vienna, 878 Austria: R Foundation for Statistical Computing. Retrieved from https://www.r-project.org/

879 Rutter, M., \& Yule, W. (1970). Neurological aspects of intellectual retardation and specific 880 reading retardation. In M. Rutter, J. Tizard, \& K. Whitmore (Eds.), Education, health and 881 behavior (pp. 54-74). London, England: Longman. 
882 Satz, P., \& Fletcher, J. M. (1987). Left-handedness and dyslexia: An old myth revisited.

883 Journal of Pediatric Psychology, 12(2), 291-298. doi:https://doi.org/10.1093/jpepsy/12.2.291

884 Somers, M., Aukes, M. F., Ophoff, R. A., Boks, M. P., Fleer, W., de Visser, K. C., ...

885 Sommer, I. E. (2015). On the relationship between degree of hand-preference and degree of

886 language lateralization. Brain and Language, 144, 10-15.

887 doi:https://doi.org/10.1016/j.bandl.2015.03.006

888 Somers, M., Neggers, S. F., Diederen, K. M., Boks, M. P., Kahn, R. S., \& Sommer, I. E. 889 (2011). The measurement of language lateralization with functional transcranial Doppler and 890 functional MRI: A critical evaluation. Frontiers in Human Neuroscience, 5, 31. doi:

891 https://doi.org/10.3389/fnhum.2011.00031

892 Somers, M., Ophoff, R. A., Aukes, M. F., Cantor, R. M., Boks, M. P., Dauwan, M., de 893 Visser, K. L., Kahn, R. S., Sommer, I. E. (2015). Linkage analysis in a Dutch population isolate 894 shows no major gene for left-handedness or atypical language lateralization. Journal of 895 Neuroscience, 35(23), 8730-8736. doi:https://doi.org/10.1523/JNEUROSCI.3287-14.2015

896 Somers, M., Shields, L. S., Boks, M. P., Kahn, R. S., Sommer, I. E. (2015). Cognitive 897 benefits of right-handedness: A meta-analysis. Neuroscience \& Biobehavioral Reviews, 51, 48898 63. doi:https://doi.org/10.1016/j.neubiorev.2015.01.003

899 Sommer, I. E., Aleman, A., Somers, M., Boks, M. P., \& Kahn, R. S. (2008). Sex 900 differences in handedness, asymmetry of the planum temporale and functional language 901 lateralization. Brain Research, 1206, 76-88.

902 doi:https://doi.org/10.1016/J.BRAINRES.2008.01.003

903 Sommer, I. E., Ramsey, N. F., Mandl, R. C., \& Kahn, R. S. (2008). Language lateralization 904 in monozygotic twin pairs concordant and discordant for handedness. Brain, 125(12), 2710905 2718. doi:https://doi.org/10.1093/brain/awf284

906 Stasinopoulos, D. M., \& Rigby, R. A. (2007). Generalized additive models for location 907 scale and shape (GAMLSS) in R. Journal of Statistical Software, 23(7), 1-46. doi:https://doi.org/ $908 \quad \underline{10.18637 / j s s . v 023 . i 07}$ 
909 Szaflarski, J. P., Binder, J. R., Possing, E. T., McKiernan, K. A., Ward, B. D., \& Hammeke, 910 T. A. (2002). Language lateralization in left-handed and ambidextrous people: fMRI data.

911 Neurology, 59(2), 238-244. doi:https://doi.org/10.1212/WNL.59.2.238

912 Torgesen, J. K., Wagner, R., \& Rashotte, C. (1999). Test of word reading efficiency

913 (TOWRE). New York, NY: Psychological Corporation.

914 Waldie, K. E., Haigh, C. E., Badzakova-Trajkov, G., Buckley, J., \& Kirk, I. J. (2013).

915 Reading the Wrong Way with the Right Hemisphere. Brain Sciences, 3(3), 1060-1075.

916 doi:https://doi.org/10.3390/brainsci3031060

917 Wechsler, D. (1999). Wechsler abbreviated scale of intelligence. New York, NY:

918 Psychological Corporation.

919 Whitehouse, A. J. O., \& Bishop, D. V. M. (2008). Cerebral dominance for language

920 function in adults with specific language impairment or autism. Brain, 131(12), 3193-3200.

921 doi:https://doi.org/10.1093/brain/awn266

922 Wilson, S. M., Bautista, A., Yen, M., Lauderdale, S., \& Eriksson, D. K. (2017). Validity

923 and reliability of four language mapping paradigms. NeuroImage, 16, 399-408.

924 doi:https://doi.org/10.1016/j.nicl.2016.03.015

925 Woodcock, R. W., McGrew, K. S., \& Mather, N. (2007). Woodcock Johnson III tests of 926 cognitive abilities. Rolling Meadows, IL: Riverside. working memory in developmental dyslexia. Journal of Neurolinguistics, 33, 67-77. 


\section{Table $\mathbf{1}$ (on next page)}

Assessment battery. 
Instrument

Wechsler Abbreviated Scale of Intelligence (WASI) (Wechsler, 1999)
Measure

Block Design

Matrices

Vocabulary

Woodcock Johnson III Tests of Cognitive Abilities (Woodcock, Verbal Comprehension McGrew, \& Mather, 2007)

NEPSY: A Developmental Neuropsychological Assessment (Korkman, Kirk, \& Kemp, 1998)
Sentence Repetition

Repetition of Nonsense Words

Oromotor Sequences

Picture Naming Test

Digit Naming Test

Sight Word Efficiency

Phonetic Decoding Efficiency

Reading Accuracy

Reading Comprehension

Reading Rate

General Communication Composite 


\section{Table 2 (on next page)}

Study variables. 


\author{
Independent Variable Dependent Variables \\ Group (DLD or TD) Handedness on the Edinburgh Handedness Inventory (EHI) \\ Quantified Hand Preference (QHP) \\ fTCD Laterality Index \\ fTCD mean \% change in left MCA blood flow during speech \\ compared to baseline \\ fTCD mean \% change in right MCA blood flow during speech \\ compared to baseline \\ $\mathrm{N}$ words produced per valid trial on $\mathrm{fTCD}$ \\ Left hemisphere dominance across laterality measures
}




\section{Table 3 (on next page)}

Descriptive results for all variables.

Means (and SDs) are presented for continuous variables, and frequencies (and percentages) are presented for categoric variables. 
TD

DLD

\section{Sample Characteristics}

$\mathrm{N}$, children

Age, years

Gender, male

\section{Cognitive Measures}

Performance IQ

Vocabulary

Verbal Comprehension

Sentence Repetition

Repetition of Nonsense Words

Oromotor Sequences

Picture Naming Test

Digit Naming Test

Sight Word Efficiency

Phonetic Decoding Efficiency
$156 \dagger$

$107 \ddagger$

$9.1( \pm 1.6) \quad 8.9( \pm 1.5)$

$56(35.9 \%) \quad 69(64.5 \%)$
$107.6( \pm 13.5) \quad 96.6( \pm 12.4)$

$$
57.5( \pm 8.6) \quad 45.3( \pm 9.7)
$$

$105.4( \pm 8.8) \quad 97.3( \pm 9.3)$

$10.2( \pm 2.7) \quad 6.8( \pm 2.9)$

$11.6( \pm 1.9) \quad 9.2( \pm 2.7)$

$3.2( \pm 1.0) \quad 1.9( \pm 0.9)$

$109.2( \pm 13.1) \quad 92.1( \pm 15.8)$

$109.8( \pm 12.6) \quad 94.1( \pm 17.1)$

$113.2( \pm 11.1) \quad 93.7( \pm 17.2)$

$111.6( \pm 13.1) \quad 93.9( \pm 14.0)$ 
Reading Accuracy

Reading Comprehension

Reading Rate

General Communication Composite

\section{Laterality Measures}

Edinburgh Handedness Inventory (EHI)

$\mathrm{N}$ children right-handed by EHI

Quantified Hand Preference (QHP)

$\mathrm{N}$ children right-handed by QHP

fTCD laterality index

Mean \% change in left MCA blood flow

Mean \% change in right MCA blood flow

$\mathrm{N}$ words produced per trial on $\mathrm{fTCD}$

$\mathrm{N}$ children with consistent left hemisphere dominance
$107.0( \pm 10.2) \quad 89.5( \pm 11.7)$

$106.4( \pm 9.5) \quad 88.8( \pm 10.1)$

$108.3( \pm 10.3) \quad 94.9( \pm 14.9)$

$86.5( \pm 15.6) \quad 62.8( \pm 22.1)$
$64.0( \pm 59.0) \quad 68.0( \pm 52.5)$

$134(85.9 \%) \quad 93(86.9 \%)$

$14.3( \pm 7.5) \quad 15.4( \pm 6.2)$

$114(73.1 \%) \quad 86(80.4 \%)$

$1.5( \pm 3.0) \quad 2.0( \pm 2.4)$

$-1.0( \pm 4.8) \quad 0.5( \pm 3.9)$

$-2.2( \pm 4.9) \quad-1.0( \pm 3.4)$

$20.7( \pm 5.1) \quad 18.7( \pm 4.7)$

$†$ Measures without complete data for all the TD children:

Picture Naming Test, $\mathrm{N}=154$; Digit Naming Test, $\mathrm{N}=155$; Reading Accuracy, $\mathrm{N}=$ 
150; Reading Comprehension, $\mathrm{N}=150$; Reading Rate, $\mathrm{N}=150$; General Communication Composite, $\mathrm{N}=136$; $\mathrm{N}$ words produced per trial on fTCD (since recording not available for all children), $\mathrm{N}=152$

$\$$ Measures without complete data for all the children with DLD:

IQ, N = 106; Picture Naming Test, N = 106; Sight Word Efficiency, N = 106; Phonetic Decoding Efficiency, $\mathrm{N}=102$; Reading Accuracy, $\mathrm{N}=98$; Reading Comprehension, $\mathrm{N}$ = 97; Reading Rate, $\mathrm{N}=98$; General Communication Composite, $\mathrm{N}=81$; $\mathrm{N}$ words produced per trial on fTCD (since recording not available for all children), $\mathrm{N}=103$ 


\section{Table 4 (on next page)}

Marginal means and $95 \% \mathrm{Cls}$ for $\mathrm{LI}$ and mean \% change in blood flow in the left and right MCAs during the period of interest compared to the baseline. 
Marginal Mean Lower 95\% CI Upper 95\% CI

TD: LI

1.52

1.07

1.96

DLD: LI

1.98

1.44

2.51

TD: Left flow, $\%$ change

$-0.84$

$-1.61$

$-0.07$

DLD: Left flow, $\%$ change

0.24

$-0.67$

1.14

TD: Right flow, \% change

$-2.14$

$-2.88$

$-1.39$

DLD: Right flow, \% change

$-1.19$

$-2.07$

$-0.32$ 


\section{Table 5 (on next page)}

Children are grouped based on evidence of left hemisphere dominance across the three laterality measures.

For each measure, 1 code for left hemisphere dominant (i.e. left-lateralised language in fTCD and right-handedness in the EHI and QHP). In fTCD, 0 codes for bilateral or right lateralised language; in the handedness measures, 0 indicates that less than half of responses were right-handed. 


\begin{tabular}{rrrrrrrrr} 
fTCD & EHI & QHP & $\begin{array}{r}\text { TD, n } \\
\text { boys }\end{array}$ & $\begin{array}{r}\text { DLD, n } \\
\text { boys }\end{array}$ & $\begin{array}{r}\text { TD, n } \\
\text { girls }\end{array}$ & $\begin{array}{r}\text { DLD, n } \\
\text { girls }\end{array}$ & $\begin{array}{r}\text { TD, n } \\
\text { children }\end{array}$ & $\begin{array}{r}\text { DLD, n } \\
\text { children }\end{array}$ \\
\hline 1 & 1 & 1 & 22 & 43 & 48 & 15 & 70 & 58 \\
1 & 0 & 1 & 2 & 3 & 0 & 0 & 2 & 3 \\
1 & 1 & 0 & 11 & 4 & 4 & 7 & 15 & 11 \\
1 & 0 & 0 & 3 & 2 & 6 & 4 & 9 & 6 \\
0 & 1 & 1 & 13 & 13 & 26 & 9 & 39 & 22 \\
0 & 0 & 1 & 2 & 1 & 1 & 2 & 3 & 3 \\
0 & 1 & 0 & 2 & 2 & 8 & 0 & 10 & 2 \\
0 & 0 & 0 & 1 & 1 & 7 & 1 & 8 & 2
\end{tabular}

1 


\section{Figure 1}

Chart showing the flow of participants through the study.

35 children were excluded because they or their twin were reported as having an ASD diagnosis; 4 were excluded because their IQ was below 70; 12 were excluded because they failed the hearing test; and 13 were excluded for other reasons, such as a medical diagnosis.

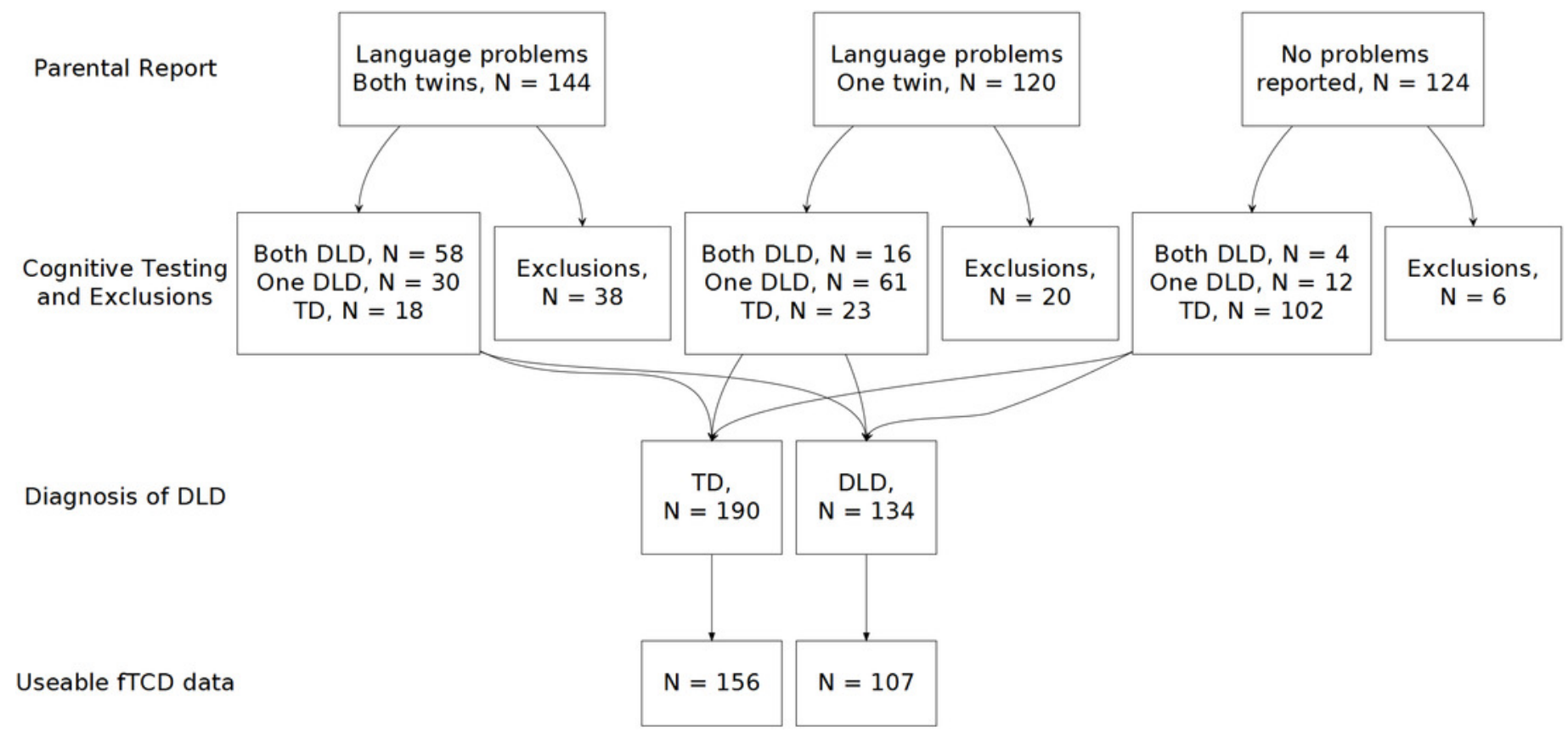


Figure 2

Pirate plot showing fTCD laterality indices (LIs) for the twins in the current study.

For comparison, we also show LIs for children using the same fTCD task reported by Groen et al. (2012). The twins are split as a function of group (TD or DLD), and all data points are shown with smoothed densities indicating the distributions in each sample. The central tendency is the mean and the intervals are Bayesian 95\% Highest Density Intervals. 


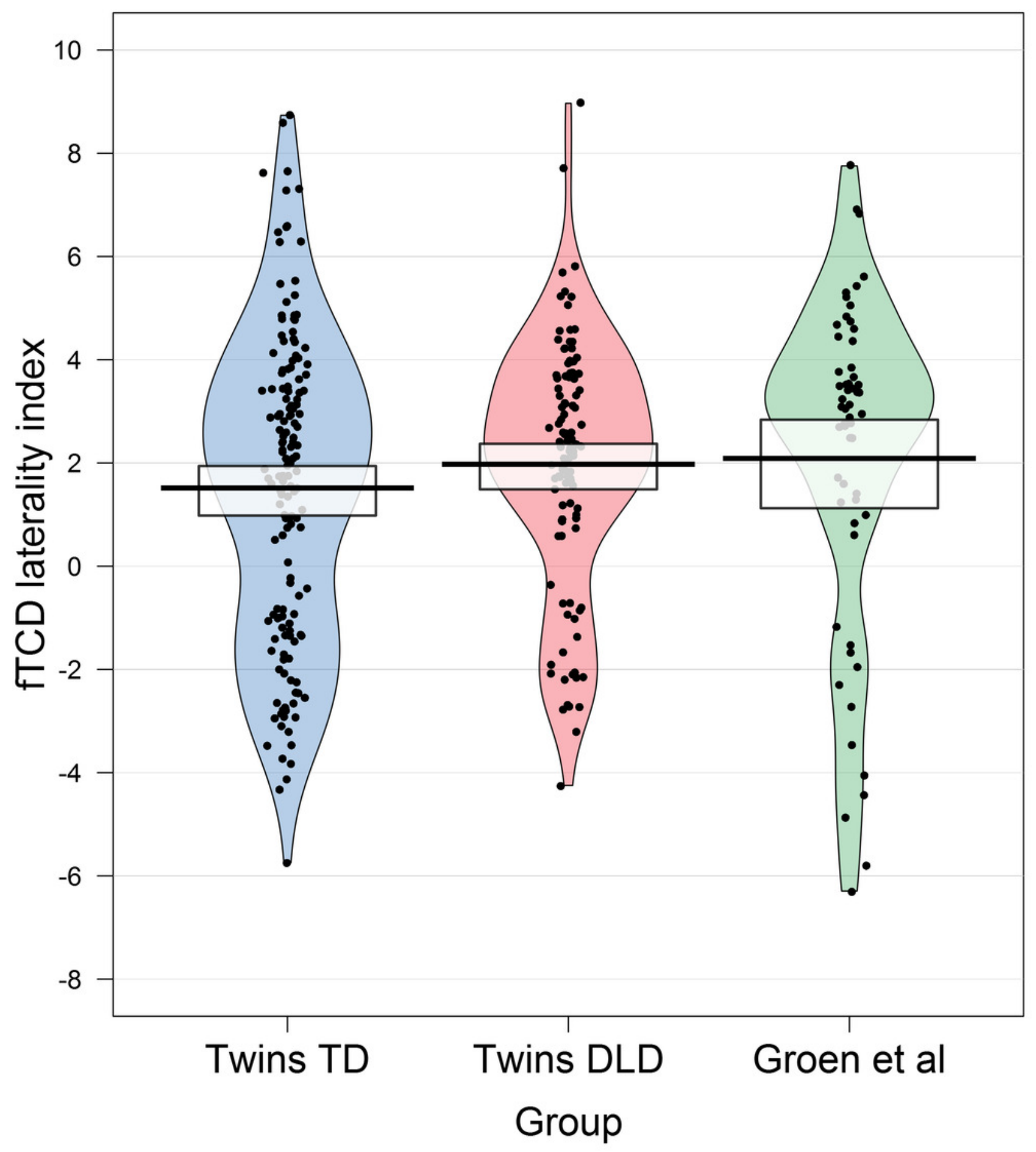




\section{Figure 3}

Plots showing the grand average curves for blood flow in the left and right MCAs for both groups.

The blue and red lines indicate blood flow in the two MCAs minus the mean baseline value, which is 100 following normalization and baseline-correction. Thus, a positive value indicates a percent increase above the mean of the baseline, and vice versa. The black line indicates the mean difference between flow in the two arteries, and therefore represents the lateralised response. The light blue area shows the period of interest during which languagerelated activity is measured. 


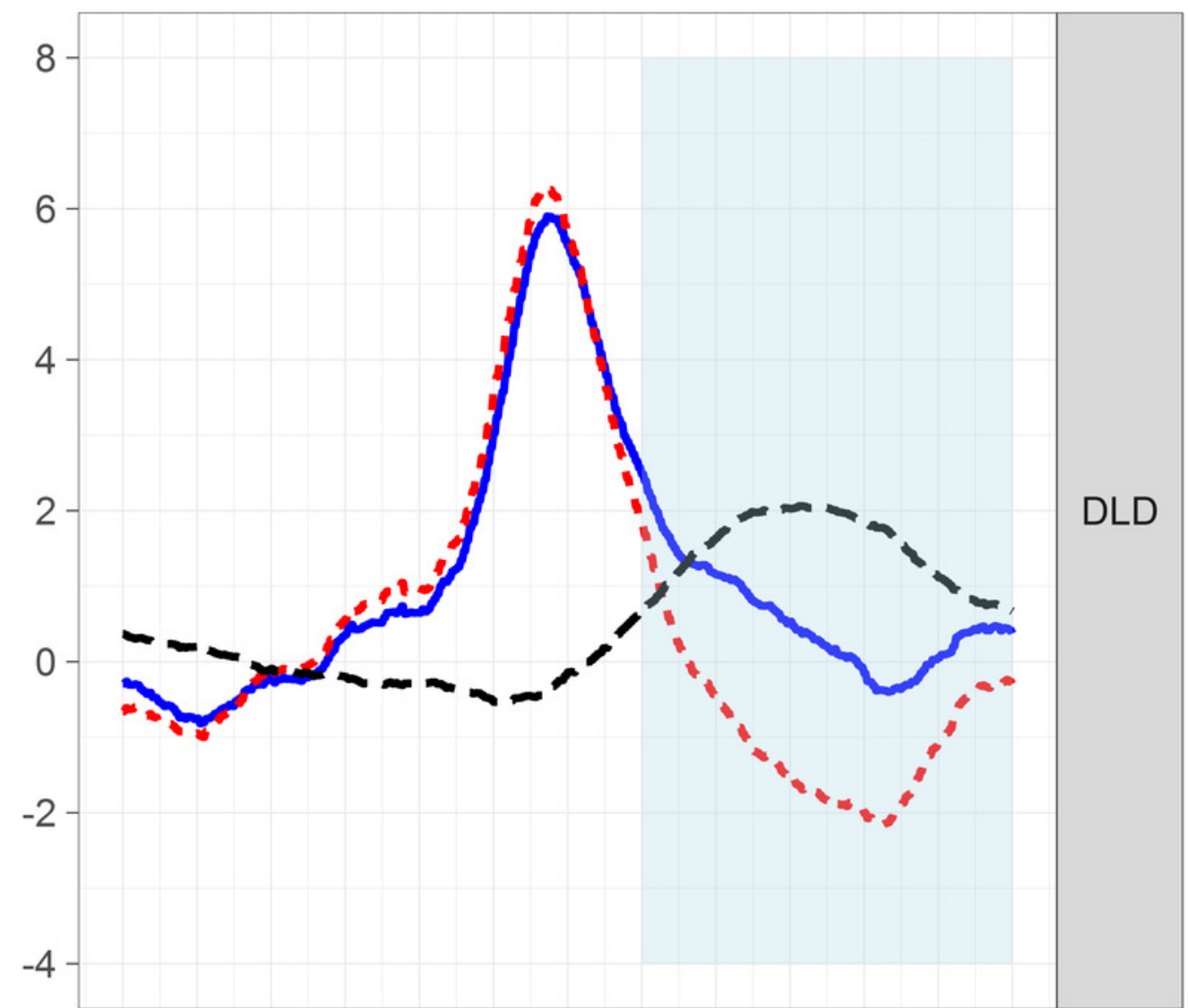

Flow

_ \% change in Left MCA

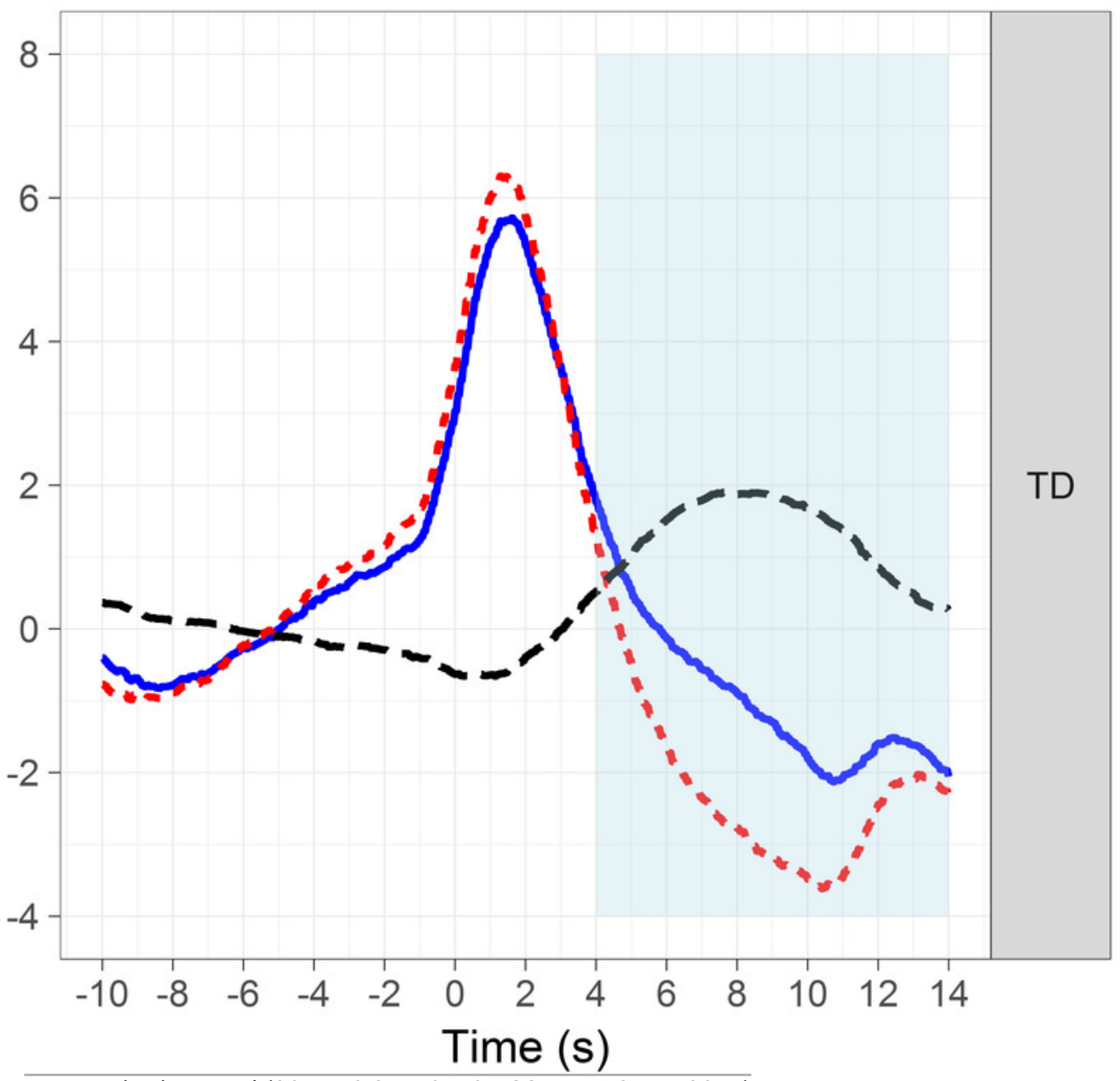
$\%$ change in - - Right MCA

_ Difference between MCAs 
Figure 4

Plot showing slopes for each group reflecting the probability of making a right hand reach to each of seven spatial positions in the QHP task.

Position 4 marks the midline. Positions 1 to 3 are to the left of the participant and positions 5 to 7 are to the right, each placed at regular intervals of 30 degrees.

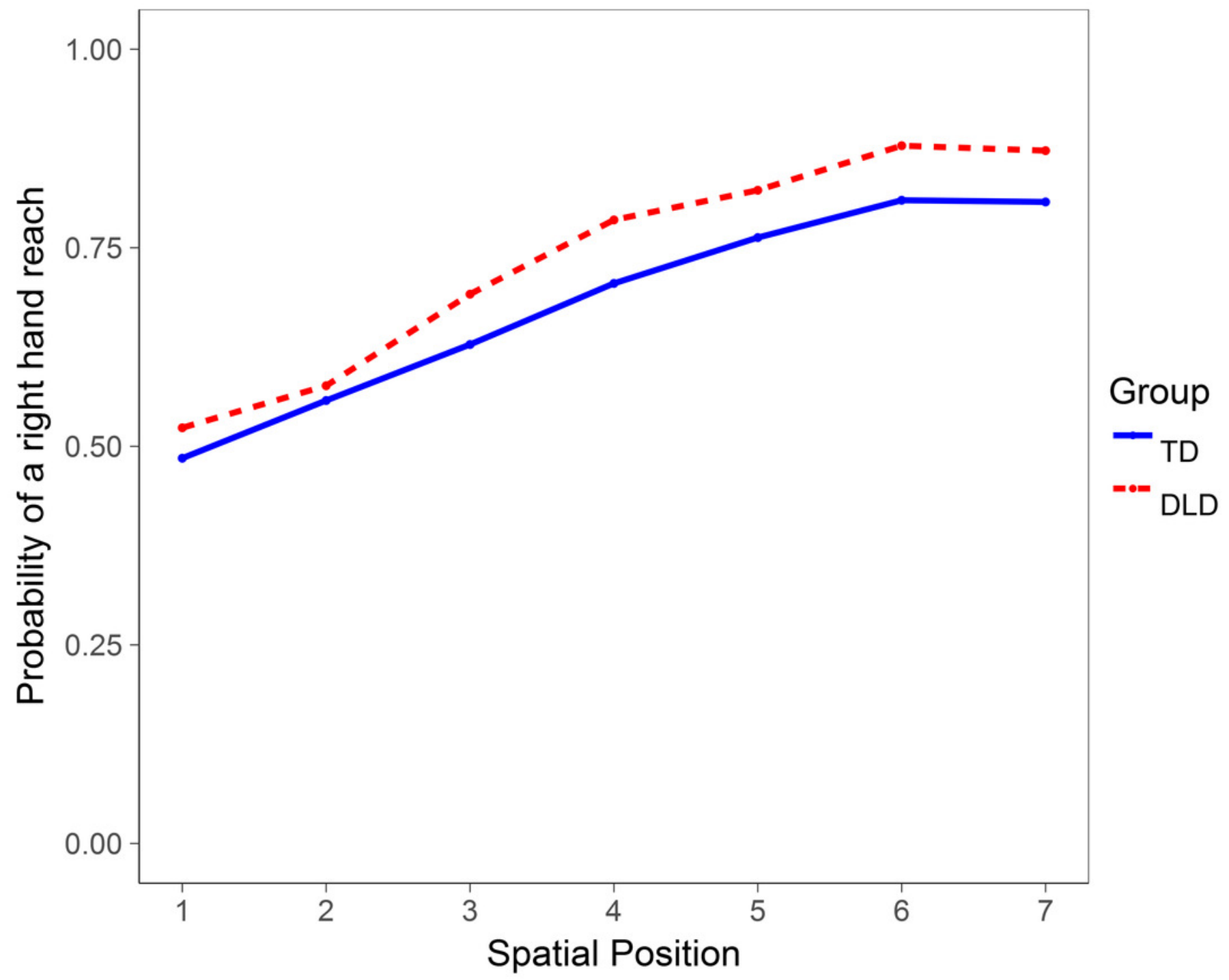

